\title{
ON THE CONSTANT IN MEŃSHOV-RADEMACHER INEQUALITY
}

\author{
SERGEI CHOBANYAN, SHLOMO LEVENTAL, AND HABIB SALEHI
}

Received 26 March 2005; Accepted 7 September 2005

The goal of the paper is twofold: (1) to show that the exact value $D_{2}$ in the MeńshovRademacher inequality equals $4 / 3$, and (2) to give a new proof of the MeńshovRademacher inequality by use of a recurrence relation. The latter gives the asymptotic estimate $\limsup _{n} D_{n} / \log _{2}^{2} n \leq 1 / 4$.

Copyright (C) 2006 Sergei Chobanyan et al. This is an open access article distributed under the Creative Commons Attribution License, which permits unrestricted use, distribution, and reproduction in any medium, provided the original work is properly cited.

\section{Introduction}

The Meńshov-Rademacher inequality deals with the estimation of

$$
D_{n}=\sup \mathbf{E} \max _{1 \leq k \leq n}\left(\sum_{l=1}^{k} \alpha_{l} \varphi_{l}\right)^{2},
$$

where sup is taken over all probability spaces $(\Omega, \mathscr{F}, P)$, all real orthonormal systems $\left(\varphi_{1}, \ldots, \varphi_{n}\right)$ on them, and all real coefficient collections $\left(\alpha_{1}, \ldots, \alpha_{n}\right)$ with $\sum_{1}^{n} \alpha_{i}^{2}=1$.

Rademacher [9] and Meńshov [7] independently proved that there exists an absolute constant $C>0$ such that for each $n \geq 2$,

$$
D_{n} \leq C \log _{2}^{2} n .
$$

A traditional proof using a bisection method (see, e.g., Doob [2] and Loève [6]) leads to the inequality

$$
D_{n} \leq\left(\log _{2} n+2\right)^{2}, \quad n \geq 2
$$

Kounias [4] used a trisection method to get a finer inequality:

$$
D_{n} \leq\left(\frac{\log _{2} n}{\log _{2} 3}+2\right)^{2}, \quad n \geq 2 \text {. }
$$

Hindawi Publishing Corporation Journal of Inequalities and Applications Volume 2006, Article ID 68969, Pages 1-7 DOI 10.1155/JIA/2006/68969 
The aim of this paper is twofold: to show that the exact starting value $D_{2}=4 / 3$ and to establish a recurrence relation which leads to a refinement of (1.4) and an asymptotic constant $\leq 1 / 4$. Note that there are several other proofs of the Meńshov-Rademacher inequality and its generalizations, see, for example, Somogyi [10] and Móricz and Tandori [8].

Section 2 deals with the proof of $D_{2}=4 / 3$, while Section 3 is devoted to the proof of the Meńshov-Rademacher inequality with the asymptotic constant $\leq 1 / 4$. Section 4 contains alternative proofs to those results using the concept of main triangle projection, a subject which was studied in depth in Gohberg and Kreĭn [3] and Kwapień and "Pełczyński" [5].

\section{The value of $D_{2}$}

Theorem 2.1. $D_{2}=4 / 3$.

The proof of the theorem is based on the following lemma which may be of independent interest.

Lemma 2.2. Let $c>0, p_{c} \equiv c^{2} /\left(1+c^{2}\right)$, and define

$$
f(p, c)=\sup _{X \in \mathscr{A}(p, c)} \mathbf{E}\left(X \mathbf{1}_{X>-c}\right), \quad p_{c} \leq p<1,
$$

where

$$
\mathscr{A}(p, c)=\left\{X \in L_{0}(\Omega, \mathscr{F}, P): \mathbf{E}(X)=0, \mathbf{E}\left(X^{2}\right)=1, P(X>-c)=p\right\}
$$

Then

$$
f(p, c)=\sqrt{p(1-p)}
$$

Proof of Lemma 2.2. To show that the left-hand side is greater than or equal to right-hand side, we observe that $\mathbf{E}\left(X_{p} \mathbf{1}_{X_{p}>-c}\right)=\sqrt{p(1-p)}$, where the distribution of $X_{p} \in \mathscr{A}(p, c)$ is given by

$$
p=P\left(X_{p}=\sqrt{\frac{(1-p)}{p}}\right)=1-P\left(X_{p}=-\sqrt{\frac{p}{(1-p)}}\right) .
$$

To see that the left-hand side is less than or equal to right-hand side, we define

$$
h_{p}(x)=x \cdot 1_{x>-c}-p \cdot x-\sqrt{\frac{p(1-p)}{4}} \cdot x^{2} .
$$

The maximum of $h_{p}(x)$ is achieved at $x=\sqrt{(1-p) / p}$ and at $-\sqrt{p /(1-p)}$ for the regions $x>-c$ and $x \leq-c$, respectively. We conclude that for any $X \in \mathscr{A}(p, c)$,

$$
0 \leq \mathbf{E}\left(h_{p}\left(X_{p}\right)\right)-\mathbf{E}\left(h_{p}(X)\right)=\mathbf{E}\left(X_{p} \cdot \mathbf{1}_{X_{p}>-c}\right)-\mathbf{E}\left(X \cdot \mathbf{1}_{X>-c}\right) .
$$

This completes the proof of the lemma. 
Let us note also that $\mathscr{A}(p, c)$ is empty for $p<p_{c}$. Indeed, by the Chebyshev inequality, $\mathbf{E}(X)=0$ and $\mathbf{E}\left(X^{2}\right)=1$ imply $P(X \leq-c) \leq 1 /\left(1+c^{2}\right)=1-p_{c}$.

Proof of Theorem 2.1. The result follows by standard calculations from the representation

$$
D_{2}=\sup _{a^{2}+b^{2}=1, b^{2} /\left(1+3 a^{2}\right)<p<1}\left\{a^{2}+b^{2} p+2 a b \cdot \sqrt{p(1-p)}\right\} .
$$

To prove (2.7) convert an orthonormal pair $\left(\varphi_{1}, \varphi_{2}\right)$ defined on $\left(\Omega, \mathscr{F}_{F}, P\right)$ into $\left(X \equiv \varphi_{1} /\right.$ $\left.\varphi_{2}, 1\right)$. The new pair is orthonormal with respect to the measure $d P^{\prime}=\varphi_{2}^{2} d P$. Also

$$
\begin{aligned}
\mathbf{E}_{P} \max & \left\{\left(a \varphi_{1}\right)^{2},\left(a \varphi_{1}+b \varphi_{2}\right)^{2}\right\}=\mathbf{E}_{P^{\prime}} \max \left\{(a X)^{2},(a X+b)^{2}\right\} \\
& =a^{2}+b^{2} P^{\prime}(X>-b / 2 a)+2 \mathrm{ab} \cdot \mathbf{E}_{P^{\prime}}\left(X \cdot \mathbf{1}_{X>-b / 2 a}\right) \\
& \leq a^{2}+b^{2} p+2 a b \cdot f\left(p, \frac{b}{2 a}\right),
\end{aligned}
$$

where $p=P^{\prime}(X>-b / 2 a)$. Now (2.7) follows from Lemma 2.2 with $c=b / 2 a$.

\section{An induction proof of the Meńshov-Rademacher inequality}

Theorem 3.1. (i)

$$
D_{m} \leq \frac{1}{4}\left(3+\log _{2} m\right)^{2}, \quad m \geq 2 .
$$

In particular, (ii)

$$
\limsup _{m} \frac{D_{m}}{\log _{2}^{2} m} \leq \frac{1}{4}
$$

LEMma 3.2. The following recurrence relation holds true for any $n \in \mathbb{N}$ :

$$
D_{2 n} \leq D_{n}+D_{n}^{1 / 2}
$$

Proof of Lemma 3.2. We have for any $n \in \mathbb{N}$,

$$
\begin{aligned}
\max _{k \leq 2 n}\left|\sum_{1}^{k} \alpha_{i} \varphi_{i}\right|^{2} & \leq \max \left(\max _{k \leq n}\left|\sum_{1}^{k} \alpha_{i} \varphi_{i}\right|^{2},\left(\left|\sum_{1}^{n} \alpha_{i} \varphi_{i}\right|+\max _{n<k \leq 2 n}\left|\sum_{n+1}^{k} \alpha_{i} \varphi_{i}\right|\right)^{2}\right) \\
& \leq \max _{k \leq n}\left|\sum_{1}^{k} \alpha_{i} \varphi_{i}\right|^{2}+2\left|\sum_{1}^{n} \alpha_{i} \varphi_{i}\right| \max _{n<k \leq 2 n}\left|\sum_{n+1}^{k} \alpha_{i} \varphi_{i}\right|+\max _{n<k \leq 2 n}\left|\sum_{n+1}^{k} \alpha_{i} \varphi_{i}\right|^{2} .
\end{aligned}
$$

Taking expectations in (3.4) and using the Cauchy-Schwartz inequality, we come to the 
4 On the constant in Meńshov-Rademacher inequality

desired recurrence relation:

$$
D_{2 n} \leq p D_{n}+2 \sqrt{p(1-p) D_{n}}+(1-p) D_{n}=D_{n}+\sqrt{D_{n}},
$$

where $p=\sum_{1}^{n} \alpha_{i}^{2}$.

The lemma is proved.

Proof of Theorem 3.1. Lemma 3.2 implies that for any $n \in \mathbb{N}$,

$$
D_{2 n}^{1 / 2} \leq D_{n}^{1 / 2}+\frac{1}{2}
$$

Since $D_{1}=1$, this implies that for each $n \in \mathbb{N}$,

$$
D_{2^{n}}^{1 / 2} \leq 1+\frac{n}{2}
$$

Let us take now $2^{n} \leq m<2^{n+1}$. Then

$$
D_{m} \leq D_{2^{n+1}} \leq\left(1+\frac{n+1}{2}\right)^{2} \leq\left(1+\frac{\log _{2} m+1}{2}\right)^{2} .
$$

This implies the validity of Theorem 3.1.

Remark 3.3. (1) The proof of Theorem 3.1 is a refinement of that appeared in Chobanyan [1].

(2) Kounias's result mentioned in the introduction leads to $\lim \sup \left(D_{n} / \log _{2}^{2} n\right) \leq$ $(\log 2 / \log 3)^{2}$ which is larger than $1 / 4$ of Theorem 3.1 .

\section{An alternative approach: the main triangle projection}

Consider the space $\mathbf{L}\left(\mathbb{R}^{n}\right)$ of all linear operators (matrices) acting in $\mathbb{R}^{n}$. The correspondence between the operators and matrices is given by $a_{i j}=\left(A e_{j}, e_{i}\right), i, j=1, \ldots, n$. The main triangle projection $T_{n}: \mathbf{L}\left(\mathbb{R}^{n}\right) \rightarrow \mathbf{L}\left(\mathbb{R}^{n}\right)$ is a linear operator introduced as follows. For an $A \in \mathbf{L}\left(\mathbb{R}^{n}\right)$, the matrix of the operator $B=T_{n} A$ has the form $b_{i j}=a_{i j}$ if $i+j \leq n+1$ and $b_{i j}=0$ otherwise.

We assume that $\mathbb{R}^{n}$ is endowed with the Euclidean norm, and the norm in $\mathbf{L}\left(\mathbb{R}^{n}\right)$ is the usual operator norm.

Theorem 4.1. $D_{n}=\left\|T_{n}\right\|^{2}, n \in \mathbb{N}$.

Proof. Let us prove first that $\left\|T_{n}\right\|^{2} \equiv \sup _{\|A\| \leq 1}\left\|T_{n} A\right\|^{2} \leq D_{n}$. Since the orthogonal operators (and only them) are the extreme points of the unit ball of $\mathbf{L}\left(\mathbb{R}^{n}\right)$, it suffices to show that for any orthogonal operator $u \in \mathbf{L}\left(\mathbb{R}^{n}\right), \quad\left\|T_{n} u\right\|^{2} \leq D_{n}$. Let us relate with $u$ the orthonormal system $\varphi_{1}, \ldots, \varphi_{n}$ defined on $(\Omega, P)$, where $\Omega=\{1, \ldots, n\}, P(j)=1 / n, j=$ $1, \ldots, n$, as follows:

$$
\varphi_{k}(j)=\sqrt{n}\left(u e_{k}, e_{j}\right), \quad k, j=1, \ldots, n
$$


We have for any vector $\alpha=\left(\alpha_{1}, \ldots, \alpha_{n}\right) \in \mathbb{R}^{n}$ with $|\alpha|=1$,

$$
\begin{aligned}
D_{n} & \geq \mathbf{E} \max _{k \leq n}\left|\sum_{i=1}^{k} \alpha_{i} \varphi_{i}\right|^{2}=\sum_{j=1}^{n} \max _{k \leq n}\left|\sum_{i=1}^{k} \alpha_{i}\left(u e_{i}, e_{j}\right)\right|^{2} \\
& \geq \sum_{j=1}^{n}\left|\sum_{i=1}^{n-j+1} \alpha_{i}\left(u e_{i}, e_{j}\right)\right|^{2}=\left\|\left(T_{n} u\right) \alpha\right\|^{2} .
\end{aligned}
$$

Taking supremum over all orthogonal $u$ 's and $\alpha$ 's from the unit ball of $\mathbb{R}^{n}$, we get $D_{n} \geq$ $\left\|T_{n}\right\|^{2}$. To prove the inverse inequality, consider an orthonormal system $\left(\varphi_{1}, \ldots, \varphi_{n}\right) \subset$ $L_{2}(\Omega, \mathscr{F}, P)$ and any vector $\alpha=\left(\alpha_{1}, \ldots, \alpha_{n}\right) \in \mathbb{R}^{n}$ with $|\alpha|=1$.

$$
I(\alpha, \varphi) \equiv \mathbf{E} \max _{k \leq n}\left|\sum_{i=1}^{k} \alpha_{i} \varphi_{i}\right|^{2}=\sum_{k=1}^{n} \mathbf{E} \mathbf{1}_{S_{k}}\left|\sum_{i=1}^{k} \alpha_{i} \varphi_{i}\right|^{2},
$$

where $S_{k}=\left\{\omega \in \Omega\right.$ : the minimum of $l^{\prime}$ s at which $\left|\sum_{i=1}^{l} \alpha_{i} \varphi_{i}(\omega)\right|$ attains its maximum equals $k\}$. Then we have

$$
I(\alpha, \varphi)=\sup _{g} \sum_{k=1}^{n}\left[\mathbf{E} g_{k} \mathbf{1}_{S_{k}}\left|\sum_{i=1}^{k} \alpha_{i} \varphi_{i}\right|\right]^{2},
$$

where supremum is taken over all collections $g=\left(g_{1}, \ldots, g_{n}\right)$ such that $g_{k}$ 's vanish outside of $S_{k}$ and $\left\|g_{k}\right\|_{2}=1, k=1, \ldots, n$. We have further

$$
\begin{aligned}
I(\alpha, \varphi) & =\sup _{g} \sum_{k=1}^{n} \sum_{i, j=1}^{k} \alpha_{i} \alpha_{j} \mathrm{E} g_{k} \varphi_{i} \varphi_{j} \\
& =\sup _{g} \sum_{i, j=1}^{n} \sum_{k=\max (i, j)}^{n} \alpha_{i} \alpha_{j} \mathrm{E} g_{k} \varphi_{i} \varphi_{j}=\sup _{g}\left\|T_{n} A \alpha\right\|^{2},
\end{aligned}
$$

where $\left(A e_{j}, e_{i}\right)=\mathbf{E} g_{n-j+1} \cdot \varphi_{i}, i, j=1, \ldots, n$. We have

$$
\|A\|=\sup _{|\alpha|=1} \sum_{i=1}^{n}\left(\sum_{j=1}^{n} \mathbf{E} \alpha_{j} g_{n-j+1} \varphi_{i}\right)^{2}=\sup _{|\alpha|=1} \sum_{i=1}^{n}\left(\mathbf{E} f \varphi_{i}\right)^{2}=\sup _{|\alpha|=1} \mathbf{E} f^{2}=1,
$$

where $f=\alpha_{j} g_{j}$, if $\omega \in S_{j}, j=1, \ldots, n$. Therefore, (4.5) implies $D_{n} \leq\left\|T_{n}\right\|^{2}$. The theorem is proved.

The following corollary is our Theorem 2.1.

Corollary 4.2. $D_{2}=4 / 3$.

Proof. We have according to Theorem 4.1,

$$
D_{2}=\left\|T_{2}\right\|^{2}=\sup _{u}\left\|T_{2} u\right\|^{2}=\sup \left\{\left\|\left(\begin{array}{ll}
a & b \\
b & 0
\end{array}\right)\right\|^{2}: a^{2}+b^{2}=1\right\}=\frac{4}{3} .
$$


Remark 4.3. It follows from the proof of Theorem 4.1 that $D_{n}=\sup \mathbf{E}\left[\max _{j}\left(\sum_{l=1}^{j} a_{l} \varphi_{l}\right)^{2}\right]$, where the supremum is over all real orthonormal systems $\varphi_{1}, \ldots, \varphi_{n}$, where each $\varphi_{j}, j=$ $1, \ldots, n$ takes at most $n$ values, and all reals $\alpha_{1}, \ldots, \alpha_{n}$ with $|\alpha|=1$.

The following lemma establishes a finer recurrence relation than Lemma 3.2. However, the two lemmas are asymptotically equivalent.

LEMMA 4.4.

$$
D_{2 n} \leq \frac{4}{3} D_{n} \quad \text { if } D_{n} \leq 3, \quad D_{2 n} \leq D_{n}-\frac{1}{2}+\sqrt{D_{n}-\frac{3}{4}} \quad \text { if } D_{n} \geq 3 .
$$

Proof. We have for any $n \in \mathbb{N}$ :

$$
\left\|T_{2 n}\right\|=\sup \left\{\left\|\left(\begin{array}{cc}
A & T_{n} B \\
T_{n} C & 0
\end{array}\right)\right\|\right\}
$$

where the supremum runs over all matrices $A, B, C$, and $D$ in $\mathbf{L}\left(\mathbb{R}^{n}\right)$ such that $\|\left(\begin{array}{c}A \\ C\end{array}\right]$ 1. For such matrices $A, B, C$, and $D$ we check that $|u A|^{2}+\left|u T_{n} B\right|^{2} \leq\left\|T_{n}\right\|^{2}|u|^{2}$ and $|A x|^{2}+\left|T_{n} C x\right|^{2} \leq\left\|T_{n}\right\|^{2}|x|^{2}$ for all $u, x \in \mathbb{R}^{n}$. Therefore, $\left\|T_{2 n}\right\| \leq \sup \{(u, A x)+(u, F y)+$ $(v, G y): u, v, x, y \in \mathbb{R}^{n},|u|^{2}+|v|^{2} \leq 1,|x|^{2}+|y|^{2} \leq 1, A, F, G \in \mathbf{L}\left(\mathbb{R}^{n}\right),\|A\| \leq 1,|w A|^{2}+$ $|w F|^{2} \leq D_{n}|w|^{2},|A z|^{2}+|G z|^{2} \leq D_{n}|z|^{2}$ for all $\left.w, z \in \mathbb{R}^{n}\right\}$. The last supremum can easily be computed and its square equals $\sup _{a \in[0,1]}\left(D_{n}-a / 2+\sqrt{D_{n} a-3 a^{2} / 4}\right)$. Hence, $D_{2 n} \leq$ $4 / 3 D_{n}$ if $D_{n} \leq 3$ and $D_{2 n} \leq D_{n}-1 / 2+\sqrt{D_{n}-3 / 4}$ if $D_{n} \geq 3$. This completes the proof of Lemma 4.4 .

Finally, it is known that for the Hilbert matrix $\left(H_{n}(i, j)=1 /(i-j)\right.$, if $i \neq j$ and $H_{n}(i, i)=$ $0, i, j=1, \ldots, n, n \geq 2)$,

$$
\frac{\left\|T_{n} H_{n}\right\|}{\left\|H_{n}\right\|} \geq \frac{\ln n}{\pi}
$$

This along with Theorem 3.1 implies the following bilateral estimate:

$$
\frac{1}{\pi^{2} \log _{2}^{2} e} \leq \liminf \frac{D_{n}}{\log _{2}^{2} n} \leq \limsup \frac{D_{n}}{\log _{2}^{2} n} \leq \frac{1}{4}
$$

\section{Acknowledgments}

This work was supported in part by the US Civilian Research and Development Foundation Award GEMI-3328-TB-03. We want to express our gratitude to the anonymous referee for bringing to our attention the relationship between $D_{n}$ and the norm of the main triangle projection. Furthemore, the results/proofs in Section 4 are based on ideas, suggestions, and comments made by the referee.

\section{References}

[1] S. Chobanyan, Some remarks on the Men'shov-Rademacher functional, Matematicheskie Zametki 59 (1996), no. 5, 787-790, translation in Mathematical Notes 59 (1996), no. 5-6, 571-574.

[2] J. L. Doob, Stochastic Processes, John Wiley \& Sons, New York, 1953. 
[3] I. C. Gohberg and M. G. Krĕn, Theory and Applications of Volterra Operators in Hilbert Space, Izdat. "Nauka", Moscow, 1967, translated in Translations of Mathematical Monographs, vol. 24, American Mathematical Society, Province, RI, 1970.

[4] E. G. Kounias, A note on Rademacher's inequality, Acta Mathematica Academiae Scientiarum Hungaricae 21 (1970), no. 3-4, 447-448.

[5] S. Kwapień and A. Pełczyński, The main triangle projection in matrix spaces and its applications, Studia Mathematica 34 (1970), 43-68.

[6] M. Loève, Probability Theory, 2nd ed., The University Series in Higher Mathematics, D. Van Nostrand, New Jersey, 1960.

[7] D. Meńshov, Sur les séries de fonctions orthogonales, I, Fundamenta Mathematicae 4 (1923), 82 105.

[8] F. Móricz and K. Tandori, An improved Menshov-Rademacher theorem, Proceedings of the American Mathematical Society 124 (1996), no. 3, 877-885.

[9] H. Rademacher, Einige Sätze über Reihen von allgemeinen Orthogonalfunktionen, Mathematische Annalen 87 (1922), no. 1-2, 112-138.

[10] Á. Somogyi, Maximal inequalities for not necessarily orthogonal random variables and some applications, Analysis Mathematica 3 (1977), no. 2, 131-139.

Sergei Chobanyan: Muskhelishvili Institute of Computational Mathematics, Georgian Academy of Sciences, 8 Akuri Street, Tbilisi 0193, Georgia

E-mail address: chobanya@stt.msu.edu

Shlomo Levental: Department of Statistics \& Probability, Michigan State University, East Lansing, MI 48824, USA

E-mail address: levental@stt.msu.edu

Habib Salehi: Department of Statistics \& Probability, Michigan State University, East Lansing, MI 48824, USA

E-mail address: salehi@stt.msu.edu 